\title{
Diagnosis of Gallbladder Tumors
}

\author{
Kazuo Inui, Junji Yoshino and Hironao Miyoshi
}

\begin{abstract}
Gallbladder cancer is among the organs examined in mass screening for cancer using ultrasonography; the reported prevalence of gallbladder cancer in such screening of a general population was $0.011 \%$, while the prevalence of gallbladder polyps was reported as 4.3 to $6.9 \%$. Endoscopic ultrasonography is useful for the differential diagnosis of gallbladder tumors detected by mass screening, as well as for estimating the depth of tumor invasion and detecting abnormal connections between pancreatobiliary ducts. While a systematic approach leading to diagnosis by endoscopic ultrasonography is useful, recent advances of contrast-enhanced ultrasonography are expected to establish it as a new modality for early detection. At our hospital, 7 of 26 patients with abnormal connections between pancreatobiliary ducts developed gallbladder carcinoma (23.1\%), and 7 of 48 patients with gallbladder carcinoma had abnormal connections between pancreatobiliary ducts $(12.5 \%)$. Serial observation in patients with gallstones and prophylactic surgery in patients with abnormal connections between pancreatobiliary ducts are necessary.
\end{abstract}

Key words: gallbladder carcinoma, polypoid gallbladder lesions, endoscopic ultrasonography, contrastenhanced ultrasonography, anomalous connections between pancreatobiliary ducts, gallstones

(Intern Med 50: 1133-1136, 2011)

(DOI: 10.2169/internalmedicine.50.5255)

\section{Introduction}

Gallbladder tumors may be detected incidentally during routine ultrasonography in patients with or without symptoms. These tumors include a variety of histologic types, such as adenocarcinoma, adenoma, hyperplasia, cholesterol polyp, inflammatory polyp, and adenomyomatosis. Accurate diagnosis is important for avoiding unnecessary surgery. Advances in imaging modalities include endoscopic ultrasonography, multi-detector-row computed tomography, magnetic resonance imaging, contrast-enhanced ultrasonography, and endoscopic retrograde cholecystography. This review provides an update on diagnosis of gallbladder tumors.

\section{Incidence of Gallbladder Carcinoma}

In Japan, gastric cancer was the leading cause of cancerrelated deaths until 1999, when lung cancer took the lead. Biliary tract cancer has been reported by the Ministry of Health, Labor and Welfare (1) to be the sixth leading cause of cancer-related deaths in Japan, where 16,841 people died of biliary tract cancer in 2007. Deaths per year from biliary tract cancer are increasing steadily, now numbering 3.8 times higher than in 1975, when 4,484 people died of biliary tract cancer. Early detection of gallbladder cancer is an urgent goal.

\section{Gallbladder Tumors in Mass Screening}

In Japan, mass screening for digestive cancer using ultrasonography has been provided through-out the country. The gallbladder is one of the organs examined. Mihara et al (2) reported the detection rate for gallbladder cancer in mass screening of 1,306,947 persons as $0.011 \%$ (143 cases); bile duct cancer, $0.003 \%$ (39 cases); and pancreatic cancer, $0.008 \%$ (114 cases). In a mass survey by our affiliated institution, detection rates of gallbladder carcinoma, gallstone, and cholesterol polyp of the gallbladder were $0.01 \%, 4.1 \%$, and $9.1 \%$ respectively among 43,348 persons (unpublished data). The detection rate of gallbladder carcinoma was similar in both studies.

In healthy populations, the prevalence of gallbladder polyps was reported as $4.3 \%$ in Denmark (3); $5.9 \%$ in Ja- 
pan (4); and $6.9 \%$ in Taiwan (5). Polypoid gallbladder lesions include a variety of pathologic types. Benign lesions are divided into two types, true tumors and pseudotumors. Pseudotumors include cholesterol polyps, inflammatory polyps, and hyperplasia. True tumors, which include adenomas and malignant lesions, should be treated by surgical resection, while pseudotumors can be observed serially. Kubota et al. (6) reported that $57 \%$ of cholesterol polyps measured less than $10 \mathrm{~mm}$, but $75 \%$ of adenomas and $13 \%$ of cancers were smaller than $10 \mathrm{~mm}$. Those authors concluded that the lesion number or size alone failed to predict histologic nature of polyps detected by ultrasonography.

\section{High Risk for Gallbladder Carcinoma}

Early detection prior to symptoms is the most important step in improving the prognosis of gallbladder carcinoma. One approach is to use certain modalities in all patients, as in mass screening. Another method is to perform periodic evaluations in patients with factors indicating a high risk of gallbladder cancer. Identification of individuals at high risk for gallbladder cancer thus is necessary to perform efficient screening.

High-risk individuals for gallbladder carcinoma have been identified as those with congenital dilation of the common bile duct, abnormal connections between pancreatobiliary ducts, reflux of pancreatic juice into the bile duct, or gallstones, as well as persons belonging to a familial gallbladder cancer kindred (7-9). Gallstones and abnormal connections between pancreatobiliary ducts and gallstones are two very important risk factors for gallbladder cancer. At our hospital, 7 of 26 patients with abnormal connections between pancreatobiliary ducts developed gallbladder cancer (23.1\%), while 7 of 48 patients with gallbladder carcinoma had an abnormal connection between pancreatobiliary ducts $(12.5 \%)$.

\section{Differential Diagnosis of Gallbladder Tumors}

\section{Multi-detector-row computed tomography}

Contrast-enhanced computed tomography can disclose the hemodynamics of gallbladder carcinoma, and it is a useful method for differential diagnosis in cases where a mass has replaced the gallbladder or thickened the gallbladder wall (10). Recently, the usefulness of multi-detector-row computed tomography was reported for tumor staging in gallbladder carcinomas (11). Although conventional computed tomography was not helpful in the differentiation when gallbladder polyps were smaller than $10 \mathrm{~mm}$ in diameter, recent advances in multi-detector-row computed tomography have increased its accuracy rate for the differential diagnosis of gallbladder polyps. Furukawa et al (12) reported that computed tomography generally shows polypoid gallbladder carcinoma as an enhancing, intraluminal tissue mass denser than surrounding bile. These authors also re- ported sensitivity, specificity, positive predictive value, negative predictive value, and the overall accuracy in computed tomographic diagnosis of neoplastic lesions to be $88 \%$, $87 \%, 88 \%, 87 \%$, and $87 \%$, respectively. The report concluded that computed tomography can reliably identify neoplastic lesions that should be resected.

\section{Magnetic resonance imaging}

Magnetic resonance imaging has not been widely used to evaluate gallbladder diseases, having the disadvantages of poor spatial and contrast resolution. However, recent advances in instrumentation have improved the spatial and contrast resolution and also the signal-to-noise ratio (13). Yoshimitsu et al (14) reported the usefulness of dynamic magnetic resonance imaging for differentiating benign from malignant gallbladder lesions. Among polypoid masses, malignant lesions $(n=9)$ demonstrated early and prolonged enhancements, while benign lesions $(n=14)$ showed early enhancement with subsequent washout.

Development of magnetic resonance cholangiopancreatography promises easy delineation of pancreatobiliary ducts and thereby detection of abnormal connections between pancreatobiliary ducts (15) which is an important risk factor for gallbladder carcinoma.

\section{Transpapillary approach}

Percutaneous transhepatic fine-needle aspiration (16) and percutaneous transhepatic cholecystoscopy have been reported as precise diagnostic techniques in the evaluation of gallbladder polyps. In 1989, we reported the usefulness of percutaneous transhepatic cholecystoscopy for diagnostic evaluation of gallbladder polyps (17). This procedure is the most reliable method for the diagnosis of gallbladder polyps, but it is time consuming and poorly tolerated by patients.

The diagnostic accuracy of endoscopic retrograde cholangiopancreatography is not satisfactory, because this method can only show a filling defect in the gallbladder without delineating the surface of polypoid lesions. Endoscopic retrograde cholangiopancreatography usually is used for the diagnosis of tumor extension to the bile duct or for demonstrating abnormal connections of pancreatobiliary ducts, an abnormality closely linked with gallbladder carcinoma. Several efforts have been made to improve the diagnosis using the transpapillary approach. Fujita et al (18) and Foerester et al (19) reported successful cases, with the expectation that this technique would expand diagnostic and therapeutic possibilities in diseases of the gallbladder. Diagnostic endoscopic inspection and biopsy would involve a slender endoscope into the gallbladder.

Watanabe et al (20) reported the clinical usefulness and problems associated with transpapillary gallbladder biopsy and obtained specimens sufficient for histologic diagnosis in 8 of 9 cases $(88.9 \%)$. Diagnostic accuracy for malignant and benign diseases was $100 \%$ and $83.3 \%$, respectively. Inserting a sheath through the cystic duct into the gallbladder is 
difficult, and obtaining biopsy specimens is more difficult on the peritoneal side than on the hepatic side of the gallbladder. Using an improved technique, Itoi et al (21) successfully cannulated the gallbladder for biopsy via the cystic duct in 71 of $85(83 \%)$. This procedure should be accepted as a precise diagnostic modality once it can be performed more easily and safely.

\section{Endoscopic ultrasonography}

Endoscopic ultrasonography is recommended for further examination after conventional ultrasonography, because images obtained are more distinct than with conventional ultrasonography. Several studies have evaluated endoscopic ultrasonography for polypoid gallbladder lesions (22-24). Such images appear promising for distinguishing cholesterol polyps from other polyps, and the overall accuracy for differentiating neoplastic from non-neoplastic masses was reported as $91.1 \%$ (22). Endoscopic ultrasonography is highly accurate in the diagnosis of cholesterol polyp and adenomyomatosis, which represent the most common types of gallbladder polyps. This ultrasonographic method can delineate cholesterol polyps, which have a thin stalk and show homogeneous echoes (except for punctuate, highly echogenic areas) from early gallbladder carcinoma, which has a thick stalk and shows heterogeneous echoes.

Mitake et al (23) reported the effectiveness of endoscopic ultrasonography in the diagnosis of gallbladder carcinoma and determination of the extent of tumor invasion; differential diagnosis between early and advanced-stage tumors was $79.5 \%$ accurate, and the overall accuracy for tumor invasion depth was $76.9 \%$. Tumor infiltration can be detected as hypoechoic tumor invading the layers of the gallbladder wall. In patients with an adenocarcinoma, characterized as $\mathrm{T} 2$ by the tumor node metastasis classification, endoscopic ultrasonography demonstrates a hypoechoic lesion at the base of tan essentially sessile polyp invading only as deeply as the second or third hyperechoic layer of the gallbladder wall (24).

Developments of contrast agents may increase the usefulness of endoscopic ultrasonography. In 1998, Hirooka et al (25) reported that in contrast-enhanced endosonography, enhancement was observed in 11 of 12 adenocarcinoma $(91.7 \%)$ but not in adenosquamous carcinoma or cholesterol polyps. Depth of tumor invasion was assessed accurately in 11 of 14 cases $(78.6 \%)$ by conventional endoscopic ultrasonography, and in 13 of 14 cases $(92.9 \%)$ by contrastenhanced endosonography.

Endoscopic ultrasonography demonstrates connections between pancreatobiliary ducts in the parenchyma of the pancreas as clearly as endoscopic retrograde cholangiopancreatography (26). As abnormal connections between pancreatobiliary ducts are closely associated with gallbladder carcinoma, identifying these is very important for early diagnosis of cancer.

\section{Contrast-enhanced ultrasonography}

Advances in conventional ultrasonography, such as highresolution ultrasonography, have contributed to improved detection of polypoid gallbladder lesions. Accuracy of ultrasonography for diagnosis of polypoid gallbladder lesions was reported as 70 to $90 \%$. Malignancy should be considered when polypoid lesions exceed $20 \mathrm{~mm}$ in diameter, are solitary, sessile or associated with gallstones. Color Doppler ultrasonography has been reported to be useful in the evaluation of malignant lesions. Hirooka et al (27) reported that in cancerous gallbladder polyps, the color signal pattern was diffuse, becoming linear at the base. Velocity and the resistance index were $39.0 \pm 12.4 \mathrm{~cm} / \mathrm{s}$ and $0.62 \pm 0.12$, respectively, which was significantly different from control measurements. In combination with conventional ultrasonography, color Doppler ultrasonography could be useful in distinguishing benign from malignant gallbladder masses.

Recently developed contrast agents may refine diagnosis by this technique. In 2007, Hattori et al (28) reported the usefulness of contrast-enhanced ultrasonography using a galactose-based contrast agent (Levovist, Nippon Schering, Japan) for differential diagnosis of polypoid gallbladder les ions. They examined contrast-enhancement patterns and time-intensity curves. Contrast enhancement patterns were classified as linear, scattered, diffuse, or branched. When diffuse and branched types were considered indicative of cancer, accuracy was $84.5 \%$, sensitivity $100 \%$, and specificity $76.9 \%$. In gallbladder carcinoma, the time-intensity curve rose sooner than in other diseases as time progressed from no contrast to early-phase. In adenocarcinoma, high-intensity values persisted at $120 \mathrm{~s}$. With an intensity of 90 or greater at $120 \mathrm{~s}$ taken to indicate cancer, accuracy was $89.7 \%$, sensitivity $89.5 \%$, and specificity $89.7 \%$. The report concluded that ultrasonographic contrast-enhancement patterns show characteristic associations with pathologic findings, serving as a valuable adjunct in diagnosis of gallbladder diseases.

Recently, another new contrast agent has become available (29). Contrast-enhanced ultrasonography using perflubutane (Sonazoid, Daiichi-Sankyo, Tokyo) has been investigated in the differential diagnosis of polypoid gallbladder lesions. In gallbladder cancer, staining throughout the tumor was continuous, consistent with diffuse hypervascularity. Differences between lesion types in flow image size and convection of blood flow were observed. Flowing images, designated as an irregular rolling sign or eruption sign, appeared to be characteristic of gallbladder cancer. On the other hand, in benign gallbladder polyps, staining was scattered with the flow image being uniform and small. Therefore, contrast-enhanced ultrasonography using perflubutane should be useful in the differential diagnosis of gallbladder tumors (29).

\section{Conclusion}

Mass screening by using ultrasonography can detect gall- 
bladder abnormalities, among which early gallbladder carcinoma should be distinguished from other diseases. Further evaluation using endoscopic ultrasonography is useful, but recent advances in contrast-enhanced ultrasonography are expected to increase capabilities for early diagnosis.

Serial observation of patients with gallstones, as well as prophylactic surgery for patients with abnormal connections between pancreatobiliary ducts, are necessary.

The authors state that they have no Conflict of Interest (COI).

\section{References}

1. Ministry of Internal Affairs and Communications. Japan Statistical Yearbook, Chapter 21 Health and sanitation 21-15 Deaths and death rate by leading cause of death. http://www.stat.go.jp/english/ data/nenkan/1431-21.htm

2. Mihara S, Yoshioka R, Tamada M, et al. Update of ultrasonographic screening for gallbladder cancer in mass survey. Tan to Sui 26: 801-804, 2005 (in Japanese, Abstract in Japanese).

3. Jorgensen T, Jensen KH. Polyps in the gallbladder: A prevalence study. Scand J Gastroenterol 25: 281-286, 1990.

4. Segawa K, Arisawa T, Niwa Y, et al. Prevalence of gallbladder polyps among apparently healthy Japanese: ultrasonographic study. Am J Gastroenterol 87: 630-633, 1992.

5. Chen CY, Lu CL, Chang FY, Lee SD. Risk factors for gallbladder polyps in the Chinese population. Am J Gastroenterol 92: 20662068, 1997.

6. Kubota K, Bandai Y, Noie T, et al. How should polypoid lesions of the gallbladder be treated in the era of laparoscopic cholecystectomy? Surgery 117: 481-487, 1995.

7. Kato K, Akai S, Tominaga S, Kato I. A case-control study of biliary tract cancer in Niigata Prefecture, Japan. Jpn J Cancer Res 80: 932-938, 1989.

8. Kimura K, Ohto M, Saisho $\mathrm{H}$, et al. Association of gallbladder carcinoma and anomalous pancreatobiliary ductal union. Gastroenterology 89: 1258-1265, 1985.

9. Yamauchi S, Koga A, Matsumoto S, Tanaka M, Nakayama F. Anomalous junction of pancreaticobiliary duct without congenital choledocal cyst: a possible risk factor for gallbladder cancer. Am J Gastroenterol 82: 20-24, 1987.

10. Yun EJ, Cho SG, Park S, et al. Gallbladder carcinoma and chronic cholecystitis: differentiation with two-phase spiral CT. Abdom Imaging 29: 102-108, 2004.

11. Kim SJ, Lee JM, Lee JY. Accuracy of preoperative T-staging of the gallbladder carcinoma using MDCT. Am J Roentgenol 190: 74-80, 2008.

12. Furukawa H, Kosuge T, Shimada K, et al. Small polypoid lesions of the gallbladder. Arch Surg 133: 735-739, 1998.

13. Tseng JH, Wan YL, Hung CF, et al. Diagnosis and staging of gall- bladder carcinoma: evaluation with dynamic MR imaging. Clin Imaging 26: 177-182, 2002.

14. Yoshimitsu K, Honda H, Kaneko K, et al. Dynamic MRI of the gallbladder lesions: Differentiation of benign from malignant. JMRI 7: 696-701, 1997.

15. Sugiyama M, Baba M, Atomi Y, Hanaoka H, Mizutani Y, Hachiya J. Diagnosis of anomalous pancreastobiliary junction: Value of magnetic resonance cholangiopancreatography. Surgery 123: 391397, 1998.

16. Wu SS, Lin KC, Yeh KT. Ultrasound-guided percutaneous transhepatic fine needle aspiration cytology study of gallbladder polypoid lesions. Am J Gastroenterol 91: 1591-1594, 1996.

17. Inui $K$, Nakazawa $S$, Yoshino $J$, et al. Percutaneous cholecystoscopy. Endoscopy 21 (suppl 1): 361-364, 1989.

18. Fujita R, Hirata N, Fujita Y. Peroral cholecystoscopy. Endoscopy 21 (suppl 1): 378-380, 1989.

19. Foerester EC, Schneider MU, Matek W, Domschke W. Transpapillary cholecystoscopy. Endoscopy 21 (supple 1): 381-383, 1989.

20. Watanabe Y, Goto H, Hirooka Y, et al. Transpapillary biopsy in gallbladder disease. Gastrointest Endosc 51: 76-79, 2000.

21. Itoi $\mathrm{T}$, Sofuni A, Itokawa $\mathrm{F}$, et al. Preoperative diagnosis and management of thick-walled gallbladder based on bile cytology obtained by endoscopic transpapillary gallbladder drainage tube. Gastrointest Endosc 64: 512-519, 2006.

22. Kimura K. Diagnosis for pedunculated polypoid lesions of the gallbladder by endoscopic ultrasonography. Nippon Shokakibyo Gakkai Zasshi (Jpn J Gastroenterol) 94: 249-260, 1997 (in Japanese, Abstract in English).

23. Mitake M, Nakazawa S, Naitoh Y, et al. Endoscopic ultrasonography in diagnosis of the extent of gallbladder carcinoma. Gastrointest Endosc 36: 562-566, 1990.

24. Inui K, Nakazawa S. Diagnosis of depth of invasion of gallbladder carcinoma with endosonography. Nippon Geka Gakkai Zasshi 99: 696-699, 1998 (in Japanese, Abstract in English).

25. Hirooka Y, Naitoh Y, Goto H, et al. Contrast-enhanced endoscopic ultrasonogramphy in gallbladder diseases. Gastrointest Endosc 48: 406-410, 1998.

26. Kimoto E, Nakazawa S, Naitoh Y, et al. Value of endoscopic ultrasonography in the detection of anomalous connection of the pancreatobiliary duct. Endoscopy 23: 117-120, 1991.

27. Hirooka Y, Naitoh Y, Goto H, Furukawa T, Ito A, Hayakawa T. Differential diagnosis of gall-bladder masses using colour Doppler ultrasonography. J Gastroenterol Hepatol 11: 840-846, 1996.

28. Hattori $M$, Inui $K$, Yoshino $J$, et al. Usefulness of contrastenhanced ultrasonography in the differential diagnosis of polypoid gallbladder lesions. Nippon Shokakibyo Gakkai Zasshi (Jpn J Gastroenterol) 104: 790-798, 2007 (in Japanese, Abstract in English).

29. Hattori $M$, Inui $K$, Yoshino $J$, et al. Usefulness of contrastenhanced ultrasonography using a new contrast agent in differential diagnosis of gallbladder polypoid lesions. Ultrasound Med Biol 35 (8S): S131, 2009.

(C) 2011 The Japanese Society of Internal Medicine http://www.naika.or.jp/imindex.html 